\title{
LIMITS TO TERTIARY ASTROMETRIC COMPANIONS IN BINARY SYSTEMS
}

\author{
Matthew W. Muterspaugh, ${ }^{1}$ Benjamin F. Lane, ${ }^{2}$ S. R. Kulkarni, ${ }^{3}$ \\ Bernard F. Burke, ${ }^{2}$ M. M. Colavita, ${ }^{4}$ and M. Shao ${ }^{4}$ \\ Received 2006 May 31; accepted 2006 August 21
}

\begin{abstract}
The Palomar High-precision Astrometric Search for Exoplanet Systems (PHASES) has monitored 37 subarcsecond binary systems to determine whether their Keplerian orbits are perturbed by faint astrometric companions to either star. Software has been developed to evaluate the regions in a companion mass-period phase space in which the PHASES observations can exclude the possibility of face-on orbit perturbations. We present results for eight systems for which astrometric companions with masses as small as those of giant planets can be excluded.
\end{abstract}

Subject headings: astrometry — binaries: close — binaries: visual — methods: data analysis — planetary systems: formation

\section{INTRODUCTION}

A technique has been developed to obtain high-precision (10$20 \mu$ as) astrometry of close stellar pairs (separation less than $1^{\prime \prime}$; Lane and Muterspaugh 2004) using long-baseline infrared interferometry at the Palomar Testbed Interferometer (PTI; Colavita et al. 1999). These observations provide precise visual orbits of the binaries and allow detection of tertiary components orbiting either the primary or secondary by way of the reflex motion of the subsystem center of light.

These measurements were made at PTI as part of the Palomar High-precision Astrometric Search for Exoplanet Systems (PHASES) program. PTI is located on Palomar Mountain near San Diego, California. It was developed by the Jet Propulsion Laboratory, California Institute of Technology, for NASA, as a testbed for interferometric techniques applicable to the Keck Interferometer and other missions such as the Space Interferometry Mission $(S I M)$. It operates in the $J(1.2 \mu \mathrm{m}), H(1.6 \mu \mathrm{m})$, and $K(2.2 \mu \mathrm{m})$ bands and combines starlight from two out of three available $40 \mathrm{~cm}$ apertures. The apertures form a triangle with one $110 \mathrm{~m}$ and two $87 \mathrm{~m}$ baselines. PHASES observations use two of the three available baselines at PTI: the north-south $(110 \mathrm{~m})$ and southwest $(87 \mathrm{~m})$ baselines.

PTI has previously contributed to exoplanet search efforts through high spatial resolution visibility "imaging" of the 51 Pegasi system (Boden et al. 1998), which placed limits on the luminosity of its companion and supported the conclusion that it is substellar, and likely planetary, in nature. Here the initial detection limits of a different effort at PTI are presented, this time based on astrometric measurements of binary systems.

\section{THE PHASES EXOPLANET SEARCH}

PHASES measures the separation vectors of bright binaries that are not resolved by PTI's telescopes but are overresolved by

\footnotetext{
1 Department of Geological and Planetary Sciences, California Institute of Technology, Pasadena, CA 91125.

2 Kavli Institute for Astrophysics and Space Research, Department of Physics, Massachusetts Institute of Technology, 70 Vassar Street, Cambridge, MA 02139.

3 Division of Physics, Mathematics and Astronomy, 105-24, California Institute of Technology, Pasadena, CA 91125.

4 Jet Propulsion Laboratory, California Institute of Technology, 4800 Oak Grove Drive, Pasadena, CA 91109.
}

the interferometer itself. The high spatial resolution $(\sim 4$ mas) of the interferometer, coupled with an hour of observations, enables extremely precise measurement of the binary separation. Phase referencing is used to maintain coherence so that the full resolving power of the interferometer is applied to the astrometric measurement. The current limiting magnitude in phase-referencing mode is $K=4.5$ for equal-magnitude binaries. Work is being done to improve the sensitivity by introducing a variation in which the phase-referencing fringe tracker operates at half the current speed, to a $50 \mathrm{~Hz}$ duty cycle. The PHASES program has successfully obtained at least one night of data on 37 binaries. As of 2006 April, observations have been attempted on 209 nights, resulting in 688 astrometric measurements.

If the primary and secondary stars are the only ones present in the system, one would expect these separation vectors to evolve according to a Keplerian model. On the other hand, if additional (faint) components are present, their presence will cause perturbations to this orbit. Distant companions simultaneously orbiting both visible components will cause only very small perturbations to the observed separation vector (the differential gravitational pull is small); the PHASES observations are not sensitive to these "circumbinary" planets (also called "P-type" or planetary-type planets; Dvorak 1982). However, companions orbiting just one star of the binary can cause noticeable perturbations to the separation vectors. This configuration is similar to that outlined by the Nemesis theory (Harrison 1977), which postulated that the Sun has a stellar companion orbiting at a distance far from the planets. PHASES is a search for these "Nemesis-type" planetary systems (also called "S-type" or satellite-type planets).

The primary goal of the PHASES program is to find and characterize giant planets in close binary systems. In this case, a "close" binary is defined as one with a semimajor axis $a \lesssim 50 \mathrm{AU}$, in which one might expect binary dynamics to play a major role in system formation and evolution (see, e.g., Pfahl \& Muterspaugh 2006). The existence of such systems poses a strong challenge for models of giant planet formation. While it is possible that each of the two processes currently favored, core accretion (Lissauer 1993) and gravitational instability (Boss 2000), contribute to giant planet formation around single stars and wide binaries, simulations show that both schemes have obstacles when a second star orbits so closely that it interacts with the planet-forming circumstellar disk (Nelson 2000), truncating it in size and heating it. In five exoplanet-hosting binaries-HD 188753 (Konacki 2005), 


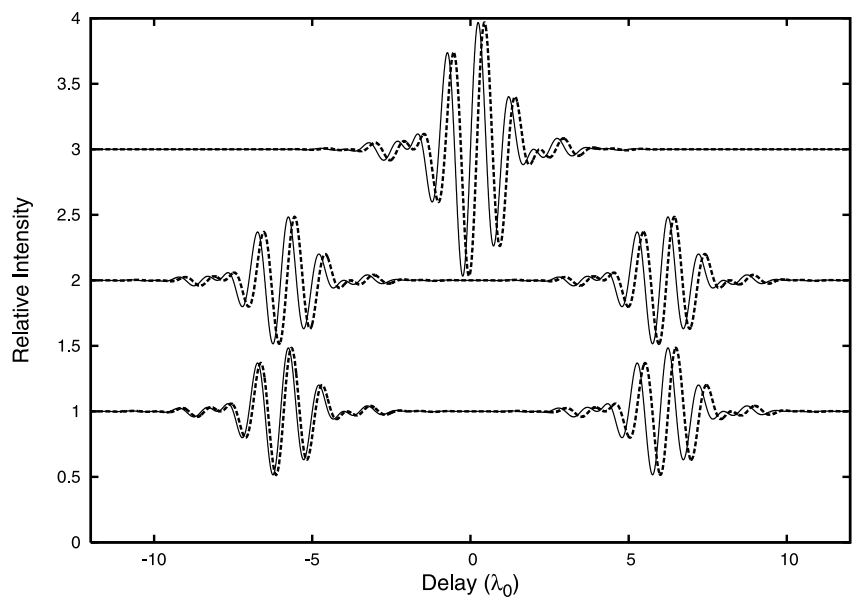

FIG. 1.-Schematic of the shift in fringe positions due to dispersion (the effect has been exaggerated for clarity). The vacuum (no dispersion) interferograms are plotted with solid lines; those dispersed by air with dotted lines. Top: Dispersion shifts the point of zero optical path difference for a star, due to the difference in air paths in each arm of the interferometer (the effective optical path difference measured as if in a vacuum). Middle: The dispersion shifts for stars of equal colors are equal and cancel; the measured separation is the same. Bottom: Stars of unequal colors are shifted by slightly different amounts by dispersion, and the resulting measured separation is different. Not shown are the shape distortions to interferograms. In a differential measurement, these cancel to first order and are insignificant at the precisions of PHASES measurements.

$\gamma$ Cephei (Hatzes et al. 2003), GJ 86 (Queloz et al. 2000), HD 41004 (Zucker et al. 2004), and HD 196885 (Chauvin et al. 2006) - the secondary star would have truncated the disks to less than $7 \mathrm{AU}$ (for systems in which the binary orbits are not fully constrained, moderate values for the eccentricities are assumed). It is possible that some of these planets formed around single stars or in wide binaries and reached their current configurations via dynamical interactions in the short-lived star clusters in which they formed (Pfahl 2005; Portegies Zwart \& McMillen 2005), although this postformation mechanism appears to be too infrequent to explain the number found.

These systems have been identified with the radial velocity (RV) method. PHASES employs astrometry, from which the companion mass can be identified without the ambiguity of the orbital inclination. Furthermore, the relative orientations of the binary and planetary orbits can be determined so that system dynamics can be studied. This effort and others specifically targeting close binaries will better determine the frequency of planets in binaries; if large, this will be strong motivation for a revolution in the theory of giant planet formation.

\section{SYSTEMATIC ERRORS}

When fit to a low-order polynomial or two-body (single) Keplerian model, the current PHASES measurements show nightto-night scatter in excess of that determined by evaluating the scatter within a given night. The timescales of this excess scatter can be evaluated by dividing observations from within a night into subsets by time and analyzing these subsets individually. It is found that within a given night the subsets agree at the level determined by the formal uncertainties obtained from the standard PHASES data reduction pipeline. Because the subsets represent observations separated by on the order of $1 \mathrm{hr}$, we conclude that the PHASES uncertainties are consistent within a night; the variations occur on night-to-night timescales.

This excess scatter may be due to either the presence of additional companions or systematic errors that occur on timescales of days or longer. In order to determine limits to tertiary compan-

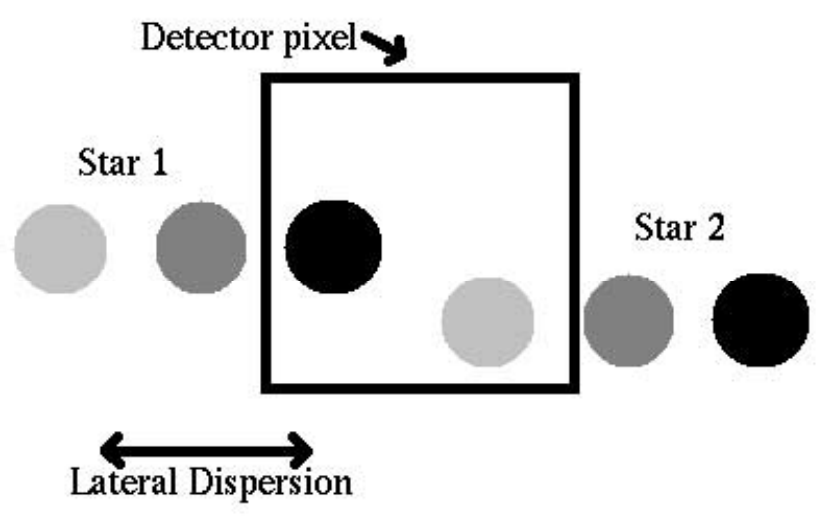

FIG. 2.- Schematic of the shift in fringe positions due to coupling of longitudinal and lateral dispersion. Due to the finite distance between stars in the image plane, the detector will sample each star differently. If the stars' spectra are dispersed in the image plane, a given detector pixel will sample different colors for each star. This is normally not a problem, as the astrometric measurement is not derived from centroiding the stars on the detector, but rather from the locations of the movable mirrors at which fringes appear on the detector from either one star or the other (each star image is the overlap of images from two telescopes). If there is additionally longitudinal dispersion, the delay location at which fringes form will be color dependent.

ions, it is assumed that any systematic errors result in true nightto-night measurement uncertainties that are related to the formal uncertainties by a multiplicative factor. Depending on the nature of potential systematics, this factor may be different for each individual system (potentially depending on contrast ratios, separations, or star color differences); it is assumed that this factor is a constant for any given system over the course of the measurements. An alternative approach would be to postulate a noise floor; this is not evaluated in the current investigation. It is assumed the uncertainties are distributed in a Gaussian manner; see $\S$ 5.4.

Potential sources of systematic instrumental errors have been identified, and efforts are being made to correct them for the next stage of PHASES observations (during the 2006-2008 seasons). The first source is due to color dispersion within the interferometer, which manifests itself in two ways. The path compensation for the geometric delay at PTI has been done with movable mirrors in air, which has a wavelength-dependent index of refraction. The fringe packets of astrophysical sources are dispersed by an amount that depends on the difference in air paths between arms of the interferometer; this changes the shape and overall location of the fringe packets. If the two stars are of differing colors, each will be dispersed by a slightly different amount, and their apparent separation will be biased; see the schematic in Figure 1. In addition, if there is dispersion in a direction perpendicular (lateral) to the light beam (due to optics acting as prisms; atmospheric differential chromatic refraction also introduces lateral dispersion, although this amount is small) or diffraction, the color of the star's light falling on the image plane detector pixel can vary with sky position. In the presence of longitudinal dispersion, the dependence of these color shifts on location within the pixel can also introduce astrometric systematics; see Figure 2. A longitudinal dispersion compensator has been commissioned for PTI, which should eliminate both systematics. A second source of potential error is drift in the "astrometric baseline" due to drifts in optical alignment, which may result in variable pupil sampling at the interferometer apertures. This is remedied by introduction of an automatic alignment system. These engineering improvements will initiate a second stage of the PHASES program, in which it is anticipated that the observational precisions will be improved by a factor of $\sim 3$. 


\section{DETECTION LIMITS}

It is anticipated that at least a portion of the excess noise is the result of systematic errors, although in some cases the presence of an astrometric companion also contributes. An approach to differentiate between these contributions is developed by recognizing that an astrometric perturbation will appear as a Keplerian wobble, whereas instrumental terms will be random; the Keplerian signature becomes a constraint on the nature of the excess scatter. An algorithm for determining the confidence levels of such a signal in the presence of instrumental scatter is described below.

\subsection{Exclusion Criteria}

The hypothesis is that model $\mathcal{A}$ correctly represents the observed system; one desires to determine the $\chi^{2}$ goodness-of-fit threshold at which a different model, $\mathcal{B}$, is less consistent with the data than $\mathcal{A}$ (for a discussion of $\chi^{2}$ as a likelihood estimator for data with Gaussian uncertainties, see, e.g., Press et al. 1992). In the present case, model $\mathcal{A}$ is the simpler single-Keplerian orbit (with seven independent parameters allowed to vary freely: $P_{1}, e_{1}, i_{1}, \omega_{1}, T_{o, 1}, \Omega_{1}$, and $a_{1}$ ), whereas model $\mathcal{B}$ is the doubleKeplerian model with additional parameters. In the case of a second "perturbation" orbit being limited to face-on circular configurations, three new free parameters will be introduced: period $P_{2}$, center-of-light semimajor axis $a_{2}$, one member of the set of the angle of periastron passage $\omega_{2}$, the epoch $T_{o, 2}$, and the longitude of the ascending node $\Omega_{2}$, which are all degenerate; the constant parameters are the other two of the previous set, the inclination $i_{2}$, which can either take value zero or $180^{\circ}$ (although nothing in between), and the eccentricity $e_{2}=0$. Extension to inclined orbits is discussed in $\S$ 4.3. A phase space can be constructed over these additional free parameters $\left(P_{2}, a_{2}\right.$, and $\left.\Omega_{2}\right)$ to evaluate which combinations result in improved or worse fits. In practice, this phase space is collapsed in the $\Omega_{2}$ dimension to the best-fit value, and $a_{2}$ is converted to tertiary companion mass.

$Q_{\mathcal{A}}$ and $Q_{\mathcal{B}}$ are defined as the number of free parameters for models $\mathcal{A}$ and $\mathcal{B}$, respectively (here, $Q_{\mathcal{A}}<Q_{\mathcal{B}}$ ), and there are $D$ independent measurements being considered (for astrometry, $D$ is twice the number of measures, as each are two dimensional). Thus, the numbers of degrees of freedom are $Z_{\mathcal{A}}=D-Q_{\mathcal{A}}$ and $Z_{\mathcal{B}}=D-Q_{\mathcal{B}}$.

In $\chi^{2}$ fitting, the best-fit model is expected to have a goodnessof-fit $\chi^{2}$ equal to the number of degrees of freedom. In the hypothesis that model $\mathcal{A}$ accurately describes the data, but that the data have excessive noise not included in their formal uncertainties, the noise excess factor is given by $\chi_{\mathcal{A}}^{2} / Z_{\mathcal{A}}$. Reweighting the formal uncertainties by the square root of this factor will result in a recomputed value of $\chi_{\mathcal{A} \text {,mod }}^{2}=Z_{\mathcal{A}}$. The hypothesis thus assumes that this new set of uncertainties correctly represents the scatter in the observations as a random process.

When introducing additional free parameters to a model, it is expected that the fit $\chi^{2}$ will decrease. This does not mean that the model fits better; rather, this must be placed in terms of the reduced $\chi_{r}^{2}=\chi^{2} / Z$. If the two models represent the data equally well, one expects

$$
\chi_{\mathcal{A}}^{2} / Z_{\mathcal{A}}=\chi_{\mathcal{B}}^{2} / Z_{\mathcal{B}}
$$

If this relationship does not hold and $\mathcal{B}$ is has the larger reduced $\chi_{r}^{2}, \mathcal{B}$ is excluded at the $N \sigma$ level for the value of $N$ that satisfies

$$
\chi_{\mathcal{A}}^{2} / Z_{\mathcal{A}}=\chi_{\mathcal{B}}^{2} /\left(Z_{\mathcal{B}}+\delta_{Q_{\mathcal{B}, N}}\right),
$$

where $\delta_{Q, N}$ is the value by which a properly normalized $\chi^{2}$ metric must be increased to find the $N \sigma$ confidence region, given
TABLE 1

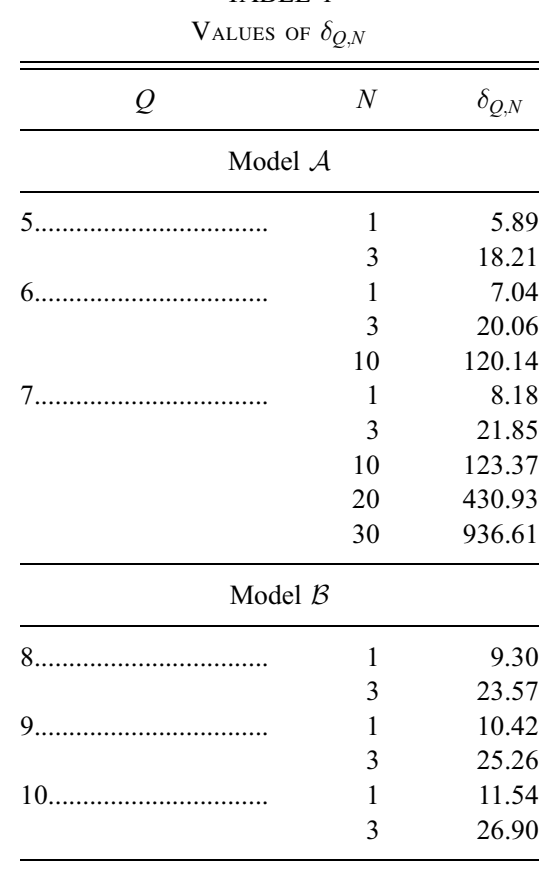

a model with $Q$ free parameters. In this case, it is the interval computed for model $\mathcal{B}$, which by assumption less accurately describes the data.

Rearrangement of terms indicates that model $\mathcal{B}$ is excluded at or beyond the $N \sigma$ level when

$$
\chi_{\mathcal{B}}^{2} \geq \frac{Z_{\mathcal{B}}+\delta_{Q_{\mathcal{B}, N}}}{Z_{\mathcal{A}}} \chi_{\mathcal{A}}^{2}
$$

\subsection{Astrometric Detection Criteria}

In this hypothesis, we accept $\mathcal{B}$ as the correct model, and look for the $\chi_{\mathcal{A}}^{2}$ level for which model $\mathcal{A}$ is excluded. Thus, we simply replace the roles of the models in equation (1):

$$
\chi_{\mathcal{A}}^{2} \geq \frac{Z_{\mathcal{A}}+\delta_{Q_{\mathcal{A}}, N}}{Z_{\mathcal{B}}} \chi_{\mathcal{B}}^{2} .
$$

Because it is model $\mathcal{B}$ for which a phase-space grid is constructed (over the extra free parameters in this more complex model), it is useful to invert this expression such that it is expressed as the contours of $\chi_{\mathcal{B}}^{2}$ for which model $\mathcal{A}$ is rejected:

$$
\chi_{\mathcal{B}}^{2} \leq \frac{Z_{\mathcal{B}}}{Z_{\mathcal{A}}+\delta_{Q_{A}, N}} \chi_{\mathcal{A}}^{2} .
$$

Table 1 lists values of $\delta_{Q, N}$ used in the following sections. The values of $\delta_{Q, N}$ can be found by iteratively solving the equation

$$
P\left(\frac{Q}{2}, \frac{\delta_{Q, N}}{2}\right)=P\left(\frac{1}{2}, \frac{N^{2}}{2}\right),
$$

where

$$
P(a, x)=\frac{\int_{0}^{x} e^{-t} t^{a-1} d t}{\int_{0}^{\infty} e^{-t} t^{a-1} d t}
$$

is the standard incomplete gamma function, equal to the probability of a $\chi^{2}$ distribution. In practice, it is often numerically better to equate $Q(a, x)=1-P(a, x)$. Thus, the $1 \sigma$ contour has probability 0.683 , and $3 \sigma$ has probability 0.9973 . 


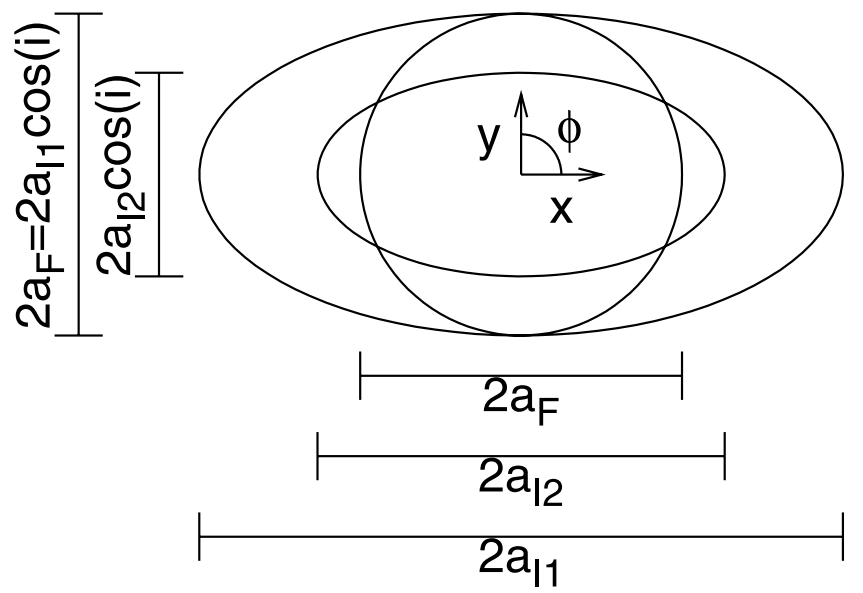

FIG. 3.- Inclined tertiary component ( perturbation) orbits ( $I 1$ and $I 2$ ) that fit observations equally as well as a face-on orbit model $(F)$. Model $I 1$ corresponds to the case of measurements with uncertainties in dimension $x$ much larger than $y$; model $I 2$ corresponds to those with equal circular uncertainty ellipses.

One must be careful how the exclusion and detection confidence levels are interpreted. This is particularly true in the case of detections; one must not forget the effect of the data sampling function or the possibility that a third model better explains the data. To claim a true astrophysical "detection," one must also satisfy the criterion that the perturbation orbit is well constrained. The exclusion criteria are more robust to such possibilities.

\subsection{Extension to Inclined Orbits}

In order for the exclusion limits to be more broadly useful, it is important to allow for the possibility of inclined orbits for the tertiary companions. The present numerical analysis is limited to face-on orbits only because of computational limitations; including a search over a range of orbital inclinations introduces two new freely varying parameters, increasing the computational time required by roughly 2 orders of magnitude. Alternatively, a procedure can be developed to relate the face-on limits to those for inclined systems.

For a given face-on system of semimajor axis $a_{F}$, one can determine the size $a_{I}$ of the inclined orbit model that results in the same $\chi^{2}$ metric; see Figure 3. Two cases are evaluated: first, when the observational uncertainty ellipses are much larger in one dimension than the other (case for model $I 1$ ), and second, when the uncertainty ellipses are circular (case for model I2). These extreme cases bound the values appropriate for PHASES data. In both cases, it is assumed that no perturbation exists and the "wide" binary orbit has been removed; the residuals are centered at $(x, y)=(0,0)$. The $\chi^{2}$ sum is divided into two terms; one summing along the axis parallel to the larger dimension of the apparent orbit of the inclined perturbation model $(x)$, and the other along the smaller dimension $(y)$ :

$$
\chi^{2}=\sum_{i=1}^{D / 2} \frac{\left[X\left(\phi\left(t_{i}\right)\right)-x_{i}\right]^{2}}{\sigma_{x}^{2}}+\sum_{i=1}^{D / 2} \frac{\left[Y\left(\phi\left(t_{i}\right)\right)-y_{i}\right]^{2}}{\sigma_{y}^{2}},
$$

where $X(\phi(t))=a \cos \phi$ and $Y(\phi(t))=a \cos i \sin \phi(i$ is the inclination of the orbit) are the perturbation orbit model values at time $t$ in each dimension ( $\phi=2 \pi t / P+\phi_{o} ; \phi_{o}$ is a phase offset), and $\sigma_{x}^{2}$ and $\sigma_{y}^{2}$ are the respective uncertainties for each dimension. There are no $x-y$ cross terms by choice of basis, explained below. Conditions for which the $\chi_{F}^{2}$ of the face-on orbit equals $\chi_{I 1}^{2}$ or $\chi_{I 2}^{2}$ of the inclined models are sought.
In the case of noncircular uncertainty ellipses, one works in a basis for which the uncertainty ellipse major axis is oriented along the $x$ axis. It is assumed the uncertainty ellipses share a single basis (their orientations do not vary) such that a $\chi^{2}$ minimizing algorithm will align the larger dimension of the apparent perturbation orbit with the large uncertainty axis (this also assumes there are no external limits placed on the longitude of the ascending node). For PHASES data, the orientations of the uncertainty ellipses vary slightly from night to night (depending on the hour angle of observations); this serves only to lessen the resulting conversion factor, and the present derivation is for the more extreme case. In the limit of large uncertainties in the $x$ direction, $|X(t)| \leq a_{I 1} \ll \sigma_{x}^{2}$ and the first term in equation (3) is unchanged and cancels on setting $\chi_{F}^{2}=\chi_{I 1}^{2}$, leaving

$$
\sum_{i=1}^{D / 2} \frac{\left[Y_{F}\left(t_{i}\right)-y_{i}\right]^{2}}{\sigma_{y}^{2}}=\sum_{i=1}^{D / 2} \frac{\left[Y_{I 1}\left(t_{i}\right)-y_{i}\right]^{2}}{\sigma_{y}^{2}} .
$$

By geometry, $Y_{F}(\phi)=a_{F} \sin \phi$ and $Y_{I 1}(\phi)=a_{I 1} \cos i \sin \phi$. By setting $a_{F}=a_{I 1} \cos i, \chi_{F}^{2}=\chi_{I 1}^{2}$. Thus, inclined orbits with semimajor axis $1 / \cos i$ times larger will fit the observations equally well as a face-on model.

For circular uncertainty ellipses, $\sigma_{x}^{2}=\sigma_{y}^{2}=\sigma^{2}$,

$$
\begin{gathered}
\sum_{i=1}^{D / 2} \frac{\left[X_{F}\left(\phi\left(t_{i}\right)\right)-x_{i}\right]^{2}}{\sigma^{2}}+\sum_{i=1}^{D / 2} \frac{\left[Y_{F}\left(\phi\left(t_{i}\right)\right)-y_{i}\right]^{2}}{\sigma^{2}} \\
=\sum_{i=1}^{D / 2} \frac{\left[X_{I 1}\left(\phi\left(t_{i}\right)\right)-x_{i}\right]^{2}}{\sigma^{2}}+\sum_{i=1}^{D / 2} \frac{\left[Y_{I 2}\left(\phi\left(t_{i}\right)\right)-y_{i}\right]^{2}}{\sigma^{2}} \\
\sum_{i=1}^{D / 2}\left[a_{F} \cos \phi\left(t_{i}\right)-x_{i}\right]^{2}+\sum_{i=1}^{D / 2}\left[a_{F} \sin \phi\left(t_{i}\right)-y_{i}\right]^{2} \\
=\sum_{i=1}^{D / 2}\left[a_{I 2} \cos \phi\left(t_{i}\right)-x_{i}\right]^{2}+\sum_{i=1}^{D / 2}\left[a_{I 2} \cos \phi\left(t_{i}\right)-y_{i}\right]^{2} .
\end{gathered}
$$

Assuming the measurements are uniformly distributed in $\phi$, and $x_{i}$ and $y_{i}$ have statistically similar distributions $p$ that are even functions (such as Gaussians), this relationship becomes

$$
\begin{gathered}
\int_{-\infty}^{\infty} \int_{0}^{2 \pi}\left(a_{F} \cos \phi-x\right)^{2} p(x) d \phi d x \\
+\int_{-\infty}^{\infty} \int_{0}^{2 \pi}\left(a_{F} \sin \phi-y\right)^{2} p(y) d \phi d y \\
=\int_{-\infty}^{\infty} \int_{0}^{2 \pi}\left(a_{I 2} \cos \phi-x\right)^{2} p(x) d \phi d x \\
\quad+\int_{-\infty}^{\infty} \int_{0}^{2 \pi}\left(a_{I 2} \sin \phi \cos i-y\right)^{2} p(y) d \phi d y \\
\int_{-\infty}^{\infty} \int_{0}^{2 \pi}\left(a_{F}^{2} \cos ^{2} \phi-2 a_{F} \cos \phi x+x^{2}\right) p(x) d \phi d x \\
\quad+\int_{-\infty}^{\infty} \int_{0}^{2 \pi}\left(a_{F}^{2} \sin ^{2} \phi-2 a_{F} \sin \phi y+y^{2}\right) p(y) d \phi d y \\
=\int_{-\infty}^{\infty} \int_{0}^{2 \pi}\left(a_{I 2}^{2} \cos ^{2} \phi-2 a_{I 2} \cos \phi x+x^{2}\right) p(x) d \phi d x \\
+\int_{-\infty}^{\infty} \int_{0}^{2 \pi}\left(a_{I 2}^{2} \sin ^{2} \phi \cos ^{2} i-2 a_{I 2} \sin \phi \cos i y+y^{2}\right) p(y) d \phi d y
\end{gathered}
$$




$$
\begin{aligned}
\int_{-\infty}^{\infty} \int_{0}^{2 \pi} & a_{F}^{2} \cos ^{2} \phi p(x) d \phi d x+\int_{-\infty}^{\infty} \int_{0}^{2 \pi} a_{F}^{2} \sin ^{2} \phi p(y) d \phi d y \\
= & \int_{-\infty}^{\infty} \int_{0}^{2 \pi} a_{I 2}^{2} \cos ^{2} \phi p(x) d \phi d x \\
& +\int_{-\infty}^{\infty} \int_{0}^{2 \pi} a_{I 2}^{2} \sin ^{2} \phi \cos ^{2} i p(y) d \phi d y
\end{aligned}
$$

where in the last step, the second terms (linear in $x$ and $y$ ) integrate to zero because $p$ is even, and the third terms cancel on the right and left sides of the equation. Finally, the remaining $x$ and $y$ integrals are common to all terms and cancel, and the $\phi$ integrals evaluate to identical values for all terms, leaving

$$
2 a_{F}^{2}=a_{I 2}^{2}\left(1+\cos ^{2} i\right) .
$$

Thus, by setting $a_{F}=a_{I 2}\left[\left(1+\cos ^{2} i\right) / 2\right]^{1 / 2}, \chi_{F}^{2}=\chi_{I 2}^{2}$.

Because orbital size is proportional to companion mass, these multiplicative factors can be directly applied to the exclusion limits for astrometric companions. To convert between the results for face-on and those including inclined models, one need multiply only by a factor between $\sim\left[2 /\left(1+\cos ^{2} i\right)\right]^{1 / 2}$ and $\sim 1 / \cos i$. The exact value of this factor depends on the distributions of observations in time and on the orientations and aspect ratios of the uncertainty ellipses.

\section{5. $\delta$ EQUULEI}

PHASES observations of the nearby binary system $\delta$ Equulei (7 Equ, HR 8123, HD 202275, ADS 14773), including an updated visual orbit, have been presented by Muterspaugh et al. (2005). The 27 observations reported there have been combined with 11 new measurements from the 2006 observing season to search for evidence of astrometric companions around either star. Figure 4 shows the regions in companion mass-period phase space for which the measurements are inconsistent with a perturbation caused by a hypothetical companion in a face-on circular orbit. Companions with masses greater than the lines plotted are inconsistent with the PHASES observations; the exclusion regions are those above the lines.

\subsection{Application of Detection Limits Procedure}

Implementation of the criteria described by equations (1) and (2) can be demonstrated on this system. The binary orbit is short enough that it is well constrained by the PHASES observations alone $\left(P_{1}<6 \mathrm{yr}\right)$; the elements of the visual orbit are not strongly covariant. Thus, all seven parameters of a visual orbit $\left(P_{1}, e_{1}, i_{1}\right.$, $\omega_{1}, T_{o, 1}, \Omega_{1}$, and $\left.a_{1}\right)$ are allowed to be varied in Keplerian model $\left(Q_{\mathcal{A}}=7\right)$. The resulting $\chi_{\mathcal{A}}^{2}$ is 1090.83 . The 38 PHASES measurements are each two-dimensional, the expected $\chi_{\mathcal{A}}^{2}$ is thus $Z_{\mathcal{A}}=$ $2 \times 38-7=69$; under the hypothesis that the single-Keplerian model is correct, $\chi_{\mathcal{A}}^{2}$ is in excess by a multiplicative factor of $1090.83 / 69=15.81$. The measurement uncertainties themselves are too small by a factor of $(15.81)^{1 / 2}=3.98$, and the median minor-axis formal uncertainty of $7.7 \mu$ as is corrected to $30.6 \mu$ as.

Model $\mathcal{B}$ is a double-Keplerian orbit given by superposition as

$$
\begin{aligned}
\boldsymbol{y}_{\mathrm{obs}}= & \boldsymbol{r}_{\mathrm{A}-\mathrm{B}}\left(P_{1}, e_{1}, i_{1}, \omega_{1}, T_{o, 1}, \Omega_{1}, a_{1}\right) \\
& +\boldsymbol{r}_{\mathrm{Ba}-\mathrm{Bb}, \mathrm{col}}\left(P_{2}, e_{2}, i_{2}, \omega_{2}, T_{o, 2}, \Omega_{2}, a_{2}\right),
\end{aligned}
$$

where, the A-B orbit is the slow, "wide" orbit and the $\mathrm{Ba}-\mathrm{Bb}$ orbit is the "narrow" perturbation orbit center-of-light motion. When converting $a_{2}$ to $M_{\mathrm{Bb}}$, assumed values for $M_{\mathrm{Ba}}=1.19 M_{\odot}$ and the system distance $d=18.386 \mathrm{pc}$ are used, and it is assumed

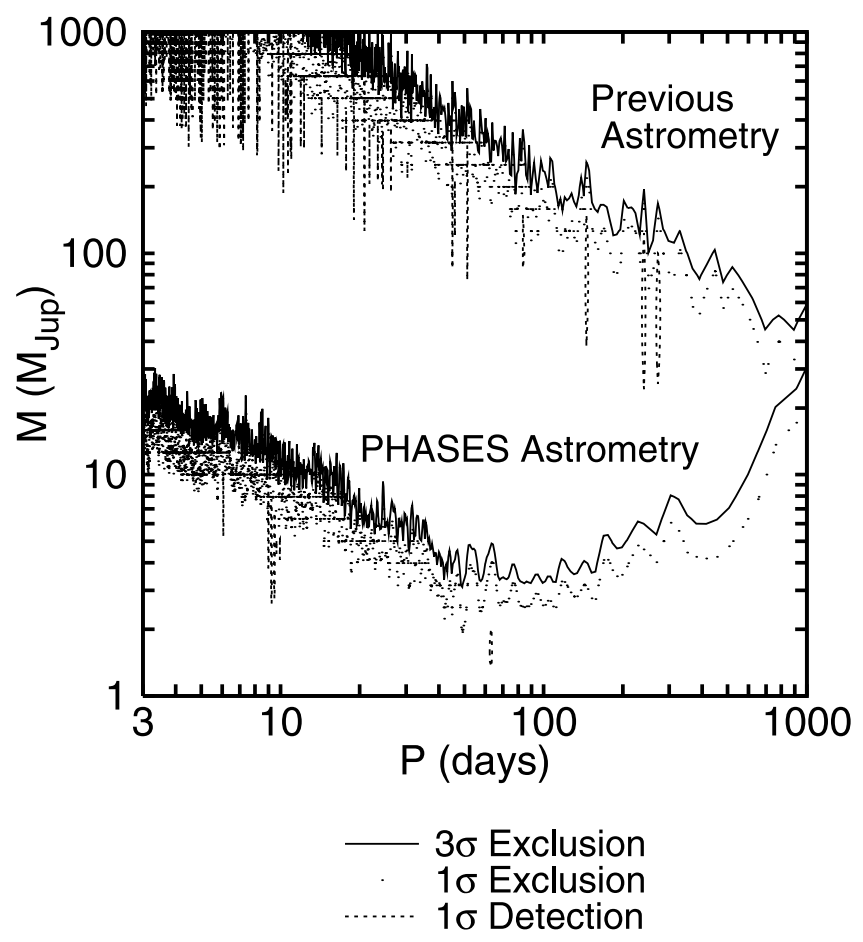

FIG. 4. $-\delta$ Equ mass-period companion phase space. Companions in the regions above the plotted exclusion curves with face-on orbits are not consistent with the observations. Companions as small as $2 M_{\mathrm{J}}$ can be ruled out by PHASES observations. The most significant detections are less than $2 \sigma$ significant and are not likely to be astrophysical in origin.

that component $\mathrm{Bb}$ is faint (i.e., the $\mathrm{Ba}-\mathrm{Bb}$ center of light is located at $\mathrm{Ba}$, and $a_{2}=a_{\mathrm{Ba}}$, corresponding to just the reflex motion of the star). In $\delta$ Equ, both stellar components $\mathrm{A}$ and $\mathrm{B}$ have mass roughly equal to $1.19 M_{\odot}$; the model is symmetric for companions to $\mathrm{A}$ or $\mathrm{B}$ and the derived limits on companion masses are identical for the two-one search eliminates companions around both stars, in this case with nearly equal mass limits.

To evaluate whether model $\mathcal{B}$ (eq. [8]) better fits the observations, the $\chi^{2}$ metric is used to evaluate the goodness of fit at each point in a grid of hypothetical companion masses and orbital periods. The grid was stepped logarithmically in companion mass from $10^{-1}$ to $10^{3} M_{\mathrm{J}}$ with step size $\log \left(M_{\mathrm{Bb}} / M_{\mathrm{J}}\right)=0.1$. The companion period was sampled at values of $P_{2}=2 f T / K$, where $f$ is an excess factor (here 3 ) for finer sampling, $T$ is the span of the observations ( $\sim 760$ days), and $K$ is a natural number up to that for which $P_{2}=3$ days; $P_{2}$ was additionally evaluated at $K=$ $1 / 2,1 / 3, \ldots, 1 / 9$.

At each grid point, the seven parameters of the wide orbit are seeded with those from the best-fit single-Keplerian (i.e., model $\mathcal{A}$ ); the perturbation orbit's parameters are seeded in the period and the (center-of-light) semimajor axis as determined by the phasespace grid. In addition, the perturbation orbit is seeded in several values of $\Omega_{2}$ (separated by $40^{\circ}$ starting at $0^{\circ}$ and increasing up to $320^{\circ}$ ), and at inclinations of either $0^{\circ}$ or $180^{\circ}$ to allow for face-on orbits with clockwise or counterclockwise motion; all combinations of these $\Omega_{2}$ and $i_{2}$ seedings are explored. The values of the other secondary orbit parameters are seeded at zero.

The model is allowed to relax from each set of initial seedings using a downhill $\chi^{2}$ fitting algorithm in which the seven parameters of the "wide" orbit and $\Omega_{2}$ in the "narrow" orbit are allowed to vary. Note that the narrow system inclination is not allowed to vary; this restricts the search to including only face-on orbits, and the inclination parameter does not add to the count of 
"free parameters" in the model. While the period and semimajor axis (and thus mass) of the perturbation orbit are not allowed to vary in the downhill fitting process, these are varied across the grid and are to be counted in the total number of model parameters. Note that with eccentricity held at zero (circular orbits) and inclinations restricted to $0^{\circ}$ and $180^{\circ}, \Omega_{2}$ is degenerate with $T_{o, 2}$ and $\omega_{2}$, and thus these need not be varied. The total number of degrees of freedom in the double-Keplerian model is thus $Q_{\mathcal{B}}=$ 10; seven are from the wide orbit, plus the period $P_{2}$, orbit phase $\Omega_{2}$, and semimajor axis $a_{2}$ (or companion mass) of the perturbing orbit. From this, $Z_{\mathcal{B}}=Z_{\mathcal{A}}-3=66$.

The $N \sigma$ exclusion regions are defined as those for which the double-Keplerian model $\chi_{\mathcal{B}}^{2}$ is greater than $1090.83 \times(66+$ $\left.\delta_{10, N}\right) / 69$. The $N \sigma$ detection regions are calculated from equation (2) as being those for which the double-Keplerian $\chi_{\mathcal{B}}^{2}$ is less than $1090.83 \times 66 /\left(69+\delta_{7, N}\right)$. The $1 \sigma\left(\chi_{\mathcal{B}}^{2}=1226\right)$ and $3 \sigma\left(\chi_{\mathcal{B}}^{2}=1469\right)$ exclusion contours in mass-period phase space are plotted in Figure 4. Also shown are the $1 \sigma$ detection contours at $\chi_{\mathcal{B}}^{2}=933$; the smallest value of $\chi_{\mathcal{B}}^{2}$ is 883 , well above the $2 \sigma$ detection criteria of $\chi_{\mathcal{B}}^{2}=864$. It is concluded these detection regions are results of statistical happenstance.

\subsection{Comparison to Previous Work}

The same procedure has been applied to the non-PHASES astrometric measurements reviewed by Muterspaugh et al. (2005); see this work for a discussion of the relative weightings of these measurements. These 170 measurements cover a much longer time span (100 yr) than the PHASES observations, but at lower precisions. In this case, the value of $T$ used to determine the companion periods sampled was chosen to be half the period of the wide orbit found in the best-fit single-Keplerian model $\left(P_{1} / 2=\right.$ $T \sim 1042)$ days. The best-fit seven-parameter single-Keplerian model results in $\chi_{\mathcal{A}}^{2}=426.5$ and $Z_{\mathcal{A}}=340-7=333$ degrees of freedom. Thus, the $1 \sigma$ and $3 \sigma$ exclusion level for $\chi_{\mathcal{B}}^{2}$ of the 10 parameter double-Keplerian model are 437.5 and 457.2 , respectively. For $P_{2}<P_{1}$, no values of $\chi_{\mathcal{B}}^{2}$ in the grid are less than 400, which is between the $2 \sigma$ and $3 \sigma$ detection thresholds (this lowest point is near $P_{2} \sim 10.25$ days and $M_{\mathrm{Bb}} \sim 800 M_{\mathrm{J}}$, which is strongly disfavored by the PHASES data).

\subsection{Inclined Orbits}

At this time, it is computationally prohibitive to search in additional parameters (for example, to add an array of seeds in inclination and allow this parameter to also vary, or to search for eccentric orbits). It is noted that there is always an orbit for which astrometry is completely insensitive to companions of any mass or period: high-eccentricity, edge-on orbits pointing directly at the observer (i.e., periastron passage within the line of sight); combination fitting with radial velocity observations lifts this problem and can be useful when contemplating universal exclusions of perturbing companions. Evaluating the phase-space grid described above using just the PHASES astrometry and a 10 parameter model requires roughly 3 weeks on a modern processor; each additional parameter is expected to increase this by roughly an order of magnitude. A need is identified for an alternative approach to the "brute-force" algorithm described here, in combination with an evaluation of its performance and reliability in comparison to the method described here.

To test the analytical conversion factor between detection limits for face-on and inclined systems of $\S 4.3$, the PHASES observations of $\delta$ Equ were reanalyzed allowing inclinations to vary within $45^{\circ}$ of face-on (both prograde and retrograde orbits were allowed) over a very limited subset of perturbation periodicities. The predicted conversion factor is between $\sqrt{4 / 3}$ and $\sqrt{2}$; in

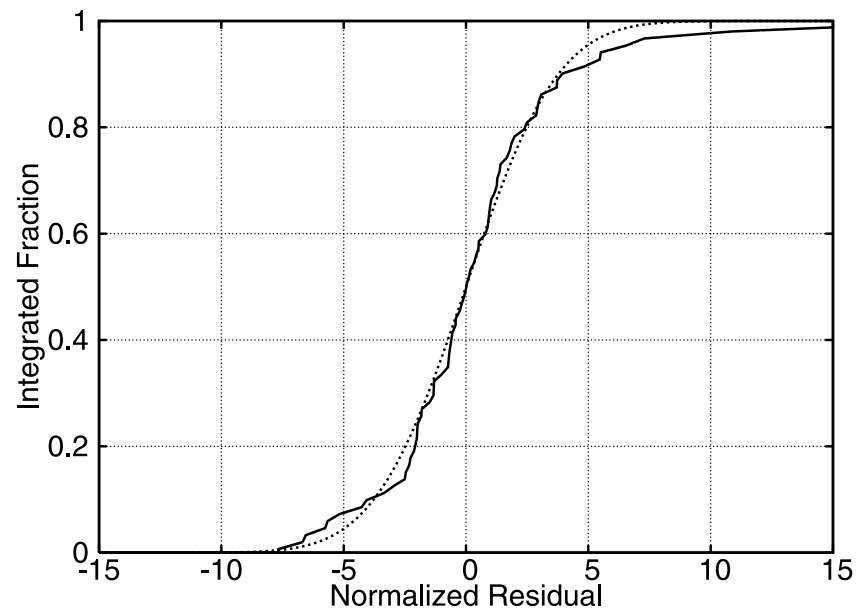

FIG. 5.-Distribution of normalized residuals for a two-body Keplerian fit to $\delta$ Equ. Also shown is the theoretical distribution for Gaussian noise.

practice, a factor of $\sim 1.35-1.4$ is appropriate for the PHASES observations.

\subsection{Fit Residuals}

Figure 5 shows the continuous (integrated) distribution function of the residuals to the two-body Keplerian fit for the $\delta$ Equ system. For each measurement, the residuals were measured along directions parallel to the formal uncertainty error ellipse's major and minor axis so that the uncertainties are not covariant. Each were then normalized by that measurement's formal uncertainty estimate. The distribution of this normalized set of residuals can now be considered to determine whether a multiplicative scale factor is an appropriate assumption for handling the observed excess scatter, in which case the residuals should have a Gaussian distribution. The continuous distribution function is fit to the integral of a Gaussian:

$$
\frac{1+\operatorname{erf}(x / \sqrt{2} a)}{2}
$$

where $a$ is a measure of the excess scatter over the formal uncertainties (the best-fit value for $a$ is $2.94 \pm 0.01$ ). A one-sided Kolmogorov-Smirnov test (see for example $\S 14.3$ of Press et al. 1992) shows the residual distribution agrees with Gaussian statistics with 95\% likelihood. The distributions match well, and the assumption of Gaussian errors and multiplicative uncertainty scale factors is valid. Treated as separate sets, the scale factors for the major-axis and minor-axis residuals are similar.

\section{KNOWN TRIPLE STARS}

This procedure is tested on two binaries that each are known to host tertiary companions, $\kappa$ Pegasi and V819 Herculis. The PHASES astrometric orbits of these triples have been previously examined (Muterspaugh et al. 2006a, 2006b); the phase-space exploration algorithm detects these perturbations and their statistical significances are now presented.

$$
\text { 6.1. } \kappa \text { Pegasi }
$$

PHASES observations of the well-known triple system $\kappa$ Pegasi (10 Peg, HR 8315, HD 206901, ADS 15281), from which the orbits of both the long and short period subsystems were determined, have been presented by Muterspaugh et al. (2006b). The 52 PHASES measurements presented there are combined with 

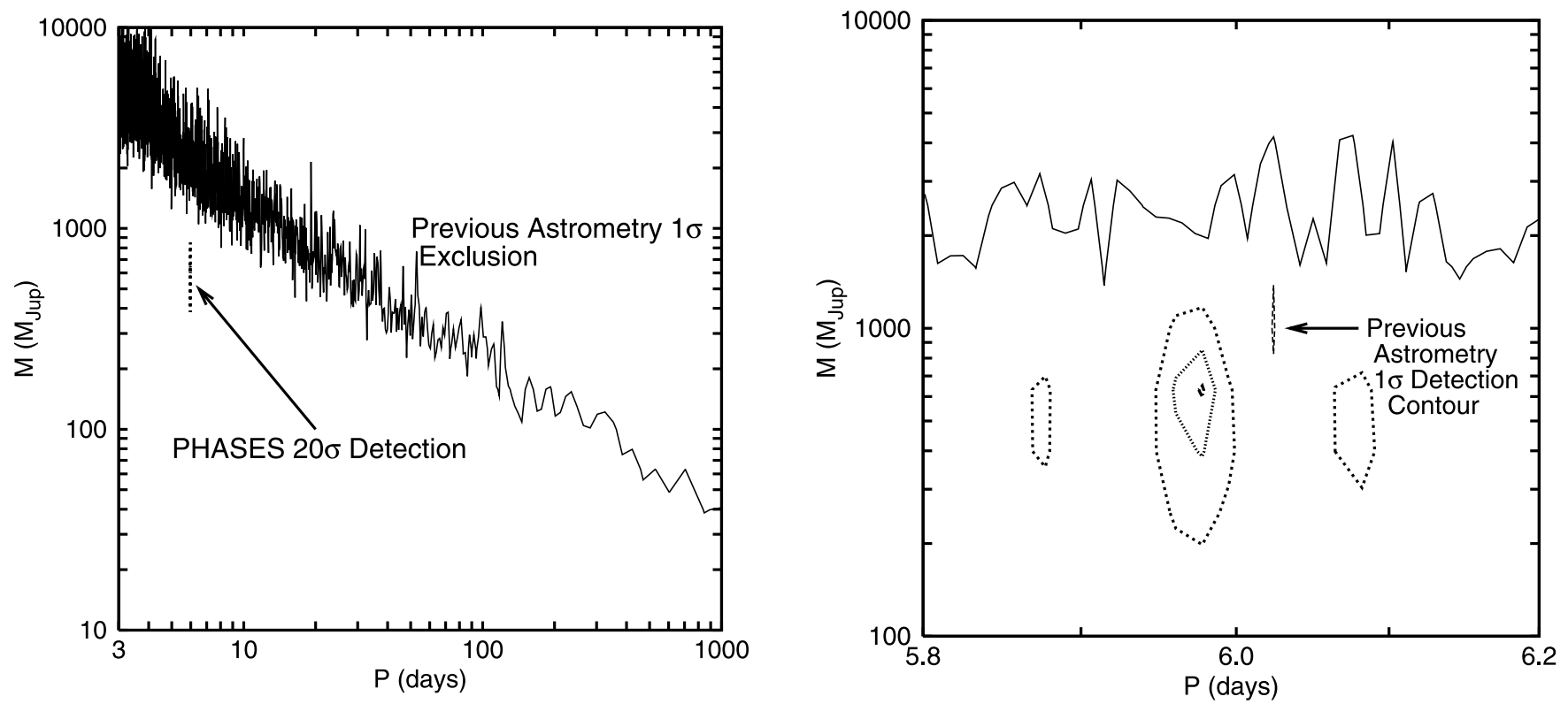

FIG. 6. $-\kappa$ Pegasi mass-period companion phase space. Left: The $1 \sigma$ exclusion contour based on previous differential astrometry data, with the $20 \sigma$ PHASES detection contour. Note that only at the 5.97 day companion period does the $20 \sigma$ PHASES contour exist. Right: The 10,20 , and $30 \sigma$ detection contours for PHASES observations of the $\kappa$ Pegasi triple star system are plotted for the limiting case of face-on Ba-Bb subsystem orbits. While the Ba-Bb subsystem is not in fact face-on, the perturbation is detected with the same periodicity as that found by RVobservations. Once this periodicity has been identified, the face-on, circular orbit constraint can be removed, and the detection becomes $106 \sigma$ significant. Also plotted is the $1 \sigma$ exclusion contour based on previous differential astrometry data. A "detection" at the $1 \sigma$ level in the previous differential astrometry is seen in the plot; however, this is likely due only to statistical happenstance.

three measurements from 2005 creating a set spanning $\sim 1046$ days. In the previous investigation, both a noise floor and multiplicative factor were explored to explain excess systematic noise; the present investigation evaluates only the latter case.

A mass-period grid was constructed similar to that in the case of $\delta \mathrm{Equ}$, differing in the following ways: the $P_{2}$ oversample factor $f$ is 1 rather than 3 , the companion masses explored ranged from $10^{0}$ to $10^{4} M_{\mathrm{J}}$, and the step size in companion mass was $\log \left(M_{\mathrm{Bb}} / M_{\mathrm{J}}\right)=0.2$ rather than 0.1 . The 424 non-PHASES astrometry data tabulated in the previous investigation were similarly evaluated over the same grid; because these span a time much longer than the wide orbit period, $T$ was set to half the bestfit wide system period $P_{1}$ (for evaluation of the PHASES data, $T$ is the time span they cover). For both the PHASES and nonPHASES data sets, the wide orbit is well constrained without strong covariances. Thus, for both cases $Q_{\mathcal{A}}=7$ and $Q_{\mathcal{B}}=10$. The companion $\mathrm{Bb}$ mass to $a_{2}$ conversion assumed a stellar mass $M_{\mathrm{Ba}}=1.662 M_{\odot}$ and distance to the system of $34.60 \mathrm{pc}$, as determined by the previous investigation. Note that this analysis is only to confirm the detection of the known 5.97 day period companion $\mathrm{Bb}$; at this time no attempt is made to search for fourth components to the system, which may require much more complicated modeling than the simple superpositioning of independent Keplerians that has been used here.

In the PHASES evaluation, the smallest value of $\chi_{\mathcal{B}}^{2}$ is found at $P_{2}=5.978$ days; in this region the step size is $\Delta P_{2} \sim 0.017$. This is consistent with the best-fit value of $P_{2}$ of $5.9714971 \pm$ $1.3 \times 10^{-6}$ days. The depth of this detection corresponds to a detection at the $31.4 \sigma$ level. Conversely, the smallest value of $\chi_{\mathcal{B}}^{2}$ for the non-PHASES data is at $P_{2}=19.14$ days with depth not quite reaching the $3 \sigma$ detection level. The smallest value with $5.9<P_{2}<6.1$ days is just barely smaller than the $1 \sigma$ threshold (near $1.1 \sigma$ ). Figure 6 plots the relevant phase-space contours.

On removing the face-on, circular-orbit criteria for the narrow orbit (seeding a $\chi^{2}$ downhill fit with the best set of parameters found in the phase-space analysis), the fit is considerably improved, $Q_{\mathcal{B}}=14$, and the second orbit improves the fit at the $106 \sigma$ level.

\subsection{V819 Herculis}

PHASES observations have previously determined the astrometric orbit of the short-period subsystem in the V819 Herculis (HR 6469, HD 157482) triple system (Muterspaugh et al. 2006a). The narrow ( $\mathrm{Ba}-\mathrm{Bb}$ ) pair of the V819 Her system is oriented edgeon and show eclipses. In that analysis, 31 measurements were reported (of which six were not used in model fitting as they were taken during eclipse of the narrow pair); one additional measurement from summer 2005 is added to this set. In the present analysis, measurements taken during eclipse are not omitted, to simulate a blind search.

When only the PHASES data are fit, the span of the measurements $(T \sim 476$ days) is much shorter than the period of the wide orbit ( $P_{1} \sim 2020$ days). Thus, $P_{1}, e_{1}$, and $a_{1}$ are strongly covariant; by fixing just one of these (here, $P_{1}=2019.787$ days) at a nominal value supported by non-PHASES observations, these covariances are lifted and the fitting algorithm converges much more rapidly. Because that parameter is not optimized for each fit, it does not count toward the number of free parameters. For the PHASES analysis, $Q_{\mathcal{A}}=6$ and $Q_{\mathcal{B}}=9$; this is not necessary when the non-PHASES observations are included, for which $Q_{\mathcal{A}}=7$ and $Q_{\mathcal{B}}=10$ as normal.

As in the case of $\kappa$ Pegasi, the mass-period phase-space grid was modified by setting the $P_{2}$ oversample factor $f$ to 1 rather than 3 , the companion masses explored ranged from $10^{0}$ to $10^{4} M_{\mathrm{J}}$, and the step size in companion mass was $\log \left(M_{\mathrm{Bb}} / M_{\mathrm{J}}\right)=0.2$ rather than 0.1 . In addition, the smallest value of $P_{2}$ sought was 1 day rather than 3. The distance is assumed to be $67.96 \mathrm{pc}$ and $M_{\mathrm{Ba}}=$ $1.43 M_{\odot}$. Again, the aim of the search is only to confirm detection of the known companion $\mathrm{Bb}$, and no attempt is made to find fourth components at this time. 


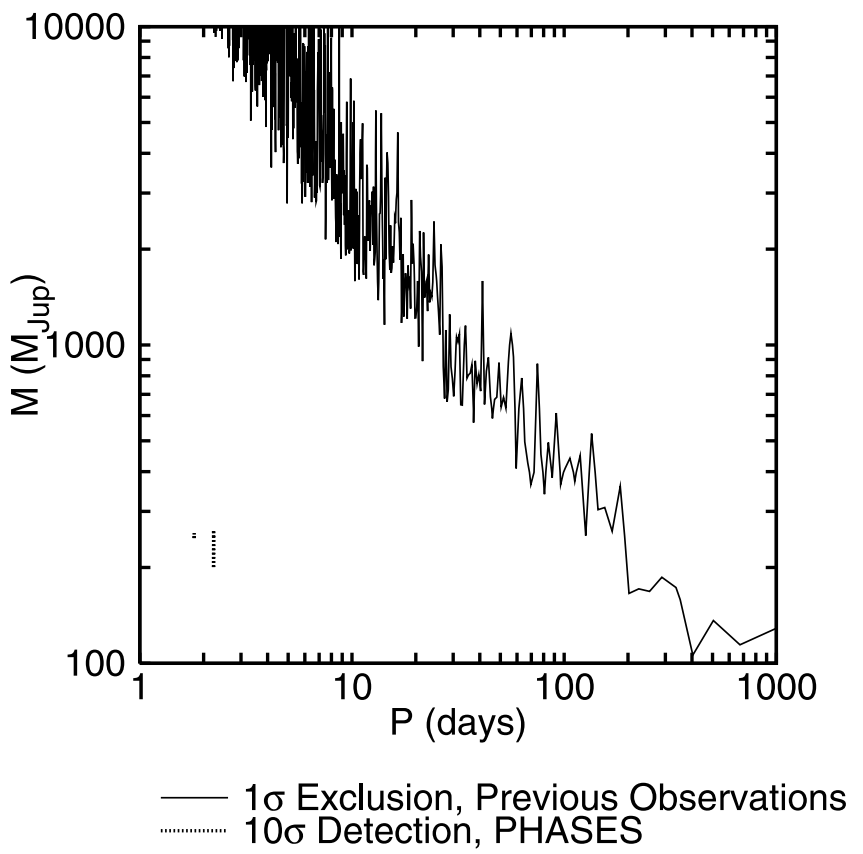

FIG. 7.-V819 Her mass-period companion phase space, showing a $10 \sigma$ significant detection in the PHASES data that is far beyond the detection threshold for previous measurements. Although the tertiary companion's orbit is in fact edge-on, the face-on code detects the wobble at the correct period. After removing the face-on orbit restraint, the double-Keplerian model is a $14.7 \sigma$ improvement over the single-Keplerian model.

Because the algorithm has been developed to specialize in face-on orbits, it is not optimally suited to analysis of the V819 Her system, for which the short-period perturbation system is observed to eclipse. However, the strength of the algorithm as a "wobble detector" is demonstrated by applying it to this system, for which it is not optimally designed. The two deepest dips in the $\chi^{2}$ function occur at periodicities of 2.2284 days $\left(\Delta P_{2} \sim\right.$ 0.0052 days; $10.5 \sigma)$ and 1.8056 days $\left(\Delta P_{2} \sim 0.0034\right.$ days; $\left.10.1 \sigma\right)$. These are the only two points at the $10 \sigma$ level. The 1.8056 day periodicity appears to be only an aliasing; the RV and eclipse photometry coincide with the (more significant) 2.2284 day periodicity (the best-fit period to all data is $P_{2}=2.2296337 \pm 1.9 \times$ $10^{-6}$ days, in agreement with the 2.2284 periodicity, to within the analysis step size). This short-period, $110 \mu$ as perturbation is readily detected, but is far beyond the ability of other astrometric measurements; with this periodicity identified, one can better refine the fit while allowing other parameters to vary, obtaining the correct orbital configuration for the short-period pair and improving the detection to the $14.7 \sigma$ level.

The 34 speckle interferometry data tabulated in the previous paper are evaluated separately (in this case $P_{1}$ is allowed to vary freely). As shown in Figure 7, the speckle observations are approximately 2 orders of magnitude away from detecting the short-period perturbation. Interestingly, the $110 \mu$ as amplitude perturbation would correspond to a planetary mass companion if only its period were several months rather than several days.

\section{7. $\beta$ CORONAE BOREALIS}

$\beta$ Coronae Borealis ("Peculiar Rosette Stone," $3 \mathrm{CrB}$, HR 5747 , HD 137909) is a binary whose primary is of spectral class F0p, part of the family of peculiar A stars with strong magnetic fields. $\beta \mathrm{CrB}$ is often grouped with $\gamma$ Equ and $\alpha^{2} \mathrm{CVn}$ as prototypes of this class. Its magnetic field has been studied extensively via measurements of Zeeman line splitting, and the inclination of its rotation axis and the angular offset of its magnetic field have been measured.

Neubauer (1944) studied the binary using RV measurements and found evidence for a third body with orbiting the primary with a period of nearly a year $\left(P_{2} \sim 320\right.$ days). Kamper et al. (1990) obtained updated RV data that did not show this perturbation. However, their analysis showed the periodicity in Neubauer's data is statistically different from $1 \mathrm{yr}$. They suggested the perturbation was real rather than instrumental, and proposed that this orbit was previously nearly face-on, somehow reorienting itself to be perfectly face-on between the two epochs. Söderhjelm (1999) concluded from Hipparcos astrometric observations that such a proposed companion cannot exist.

Forty-two PHASES measurements have been collected over a span of $\sim 804$ days. In addition, 102 non-PHASES differential astrometry measurements have been identified (when these are analyzed, $T$ is set to half the best-fit value of $P_{1}$ in the two-body single-Keplerian case). As was the case for V819 Her, $P_{1}, e_{1}$, and $a_{1}$ are strongly covariant when only PHASES data are considered ( $T \sim 804$ days vs. $P_{1} \sim 10.27$ yr). Again, $P_{1}$ is fixed at a value that is not based on the PHASES observations; $P_{1}=10.27 \mathrm{yr}$ is adopted from Tokovinin (1984) and $Q_{\mathcal{A}}=6, Q_{\mathcal{B}}=9$ (7 and 10 when non-PHASES data are included).

The mass-period grid is set up in the same manner as for $\delta$ Equ. Based on analysis of Hipparcos observations by Söderhjelm (1999), the distance is assumed to be $34.12 \mathrm{pc}(\pi=29.31 \pm$ $0.82 \mathrm{mas}$ ), and the component masses are $1.77 \pm 0.16$ and $1.21 \pm$ $0.11 M_{\odot}$. The conversion of tertiary mass to semimajor axis assumes a stellar mass of $1.21 M_{\odot}$. Radial velocity measurements are available only for one component of the binary; this prevents one from determining the masses and distance from the orbit alone.

The excess multiplicative factor is $\chi_{\mathcal{A}}^{2} / Z_{\mathcal{A}}=9.9 \sim 3.2^{2}$ for the PHASES observations. The $1 \sigma$ and $3 \sigma$ exclusion contours for both the PHASES and non-PHASES data sets are shown in Figure 8 . In both cases, the $P_{2} \sim 320$ day perturbation is excluded; for the PHASES observations, a companion of mass as small as $4 M_{\mathrm{J}}$ are excluded. The mass limits should be increased by $\sim 1.77 / 1.21 \sim 1.46$ when considering the primary star as the companion host (this estimate assumes the companion is an insignificant part of the total mass, reasonable in the case of the lower limit exclusion region). It appears that the perturbation in Neubauer's data set is not astrophysical in origin.

There are regions of the mass-period phase space for which perturbations from a tertiary companion improve the fit by $3 \sigma$ or more. The smallest value of $\chi_{\mathcal{B}}^{2}$ corresponds to a $5.2 \sigma$ detection at $P_{2} \sim 20.9$ days. When the restriction to face-on, the narrow orbit's parameters are strongly covariant and poorly constrained; for example, the formal uncertainty on the eccentricity is 0.5 , implying it is not constrained at all, and the Eulerian angles all have formal uncertainties greater than $360^{\circ}$. While the double-Keplerian model can formally improve the fit compared to the single version, the double-Keplerian model is too detailed to be properly constrained by the current observations. In order to claim a true detection, multiple criteria must be met: the double-Keplerian model must better fit the data, and the perturbation orbit must be well constrained. Thus, it is possible that the "detection" periodicities are astrophysical in nature, but the data sampling in the present set prevents conclusive study, and aliasing causes multiple periodicities to exist.

\section{13 PEGASI}

13 Pegasi (HR 8344, HD 207652) has been observed 25 times over a span of $T \sim 441$ days in the PHASES program. The span 


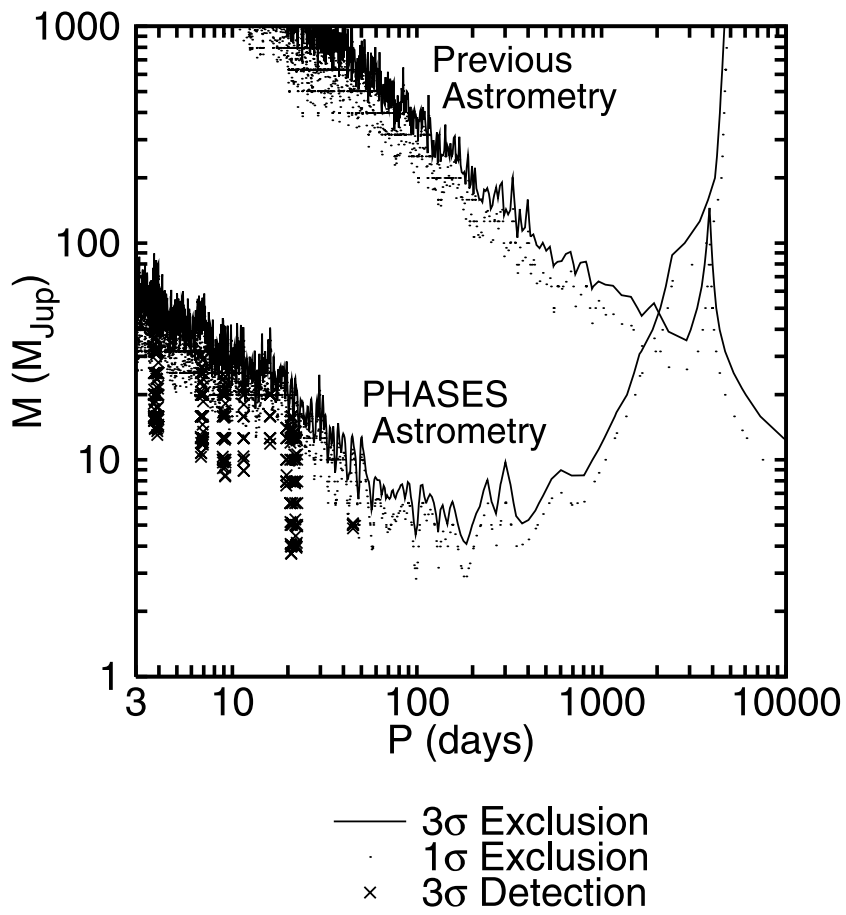

Fig. 8.-Mass-period phase space for tertiary companions to $\beta \mathrm{CrB}$, showing that a proposed massive object with period $\sim 320$ days does not exist. Some mass-period combinations do result in double-Keplerian fits that are an improvement over the single-Keplerian model, but the inner body's orbit is poorly constrained, and it is not clear whether these are astrophysical in origin.

is short compared to the binary period $\left(P_{1} \sim 26.132 \mathrm{yr}\right.$; Hartkopf et al. 1989) and again $P_{1}, e_{1}$, and $a_{1}$ are covariant; in the analysis $P_{1}$ is thus held fixed at this nominal value $\left(Q_{\mathcal{A}}=6\right.$ and $\left.Q_{\mathcal{B}}=9\right)$. Söderhjelm (1999) used Hipparcos measurements to determine the system parallax of $29.6 \pm 0.8$ mas, a total mass of $2.67 M_{\odot}$, and a photometric mass ratio $M_{\mathrm{B}} / M_{\mathrm{A}}=0.80$. Computations assume a distance of $33.8 \mathrm{pc}$ and the tertiary companion orbits the secondary at mass $1.19 M_{\odot}$ (the results can be scaled appropriately for the primary at mass $1.48 M_{\odot}$ ).

The best-fit $\chi_{\mathcal{A}}^{2}=148.8$, with $Z_{\mathcal{A}}=44$ degrees of freedom; assuming model $\mathcal{A}$ is correct, the multiplicative excess factor is $3.38 \sim 1.84^{2}$. The median formal minor axis uncertainty of $10.2 \mu$ as is thus adjusted to $18.8 \mu$ as repeatability from night to night. The mass-period grid is set up as in the case of $\delta$ Equ. Figure 9 shows that massive planets in 20 day to $3 \mathrm{yr}$ period face-on circular orbits would perturb this binary by more than the observed scatter in the PHASES data. Planets as small as $2 M_{\mathrm{J}}$ are ruled out in $\sim 4$ month period orbits. A number of regions show $1 \sigma$ consistent detections; the best of these is at only the $1.7 \sigma$ detection level.

\section{20 PERSEI}

The binary 20 Persei (HR 855, HD 17904, ADS 2200) has been observed 26 times by PHASES. At $K=4.3$ it is one of the fainter PHASES targets. The observations span $T \sim 877$ days, a small amount compared to the orbital period of $P_{1} \sim 31.528 \mathrm{yr}$. Covariances between $P_{1}, e_{1}$, and $a_{1}$ are extremely strong. Using values from Docobo et al. (2001), both $P_{1}(31.528 \mathrm{yr})$ and $e_{1}(0.753)$ are fixed $\left(Q_{\mathcal{A}}=5\right.$ and $\left.Q_{\mathcal{B}}=8\right)$. The system distance is assumed to be $72.1 \mathrm{pc}$ from the Hipparcos parallax; combining this with the orbital period and semimajor axis an average component mass of $2.1 M_{\odot}$ is computed and these values are used in converting tertiary mass to $a_{2}$. The mass-period grid is set up

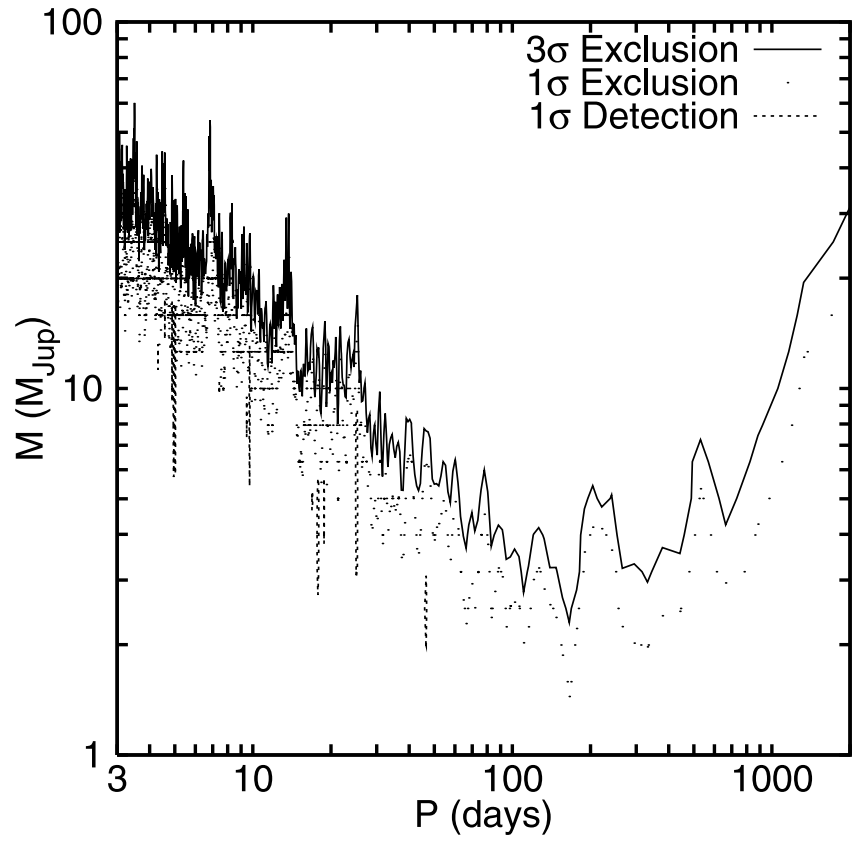

FIG. 9.-13 Pegasi mass-period companion phase space, showing that PHASES observations can rule out tertiary objects as small as $2 M_{\mathrm{J}}$. A few massperiod combinations introduce slight improvements over the single-Keplerian model, but none of these are more significant than $1.7 \sigma$ and are probably not astrophysical in origin.

following the same procedure as for $\delta$ Equ. For $20 \mathrm{Per}, \chi_{\mathcal{A}}^{2} / Z_{\mathcal{A}}=$ $6.57 \sim 2.56^{2}$ is a relatively small value. However, the average formal minor-axis uncertainty is $21 \mu$ as; coupling this with the large distance and masses implies that the tertiary detection limits are not particularly sensitive.

The PHASES measurements rule out brown dwarf mass companions in a variety of orbital periods, as shown in Figure 10.

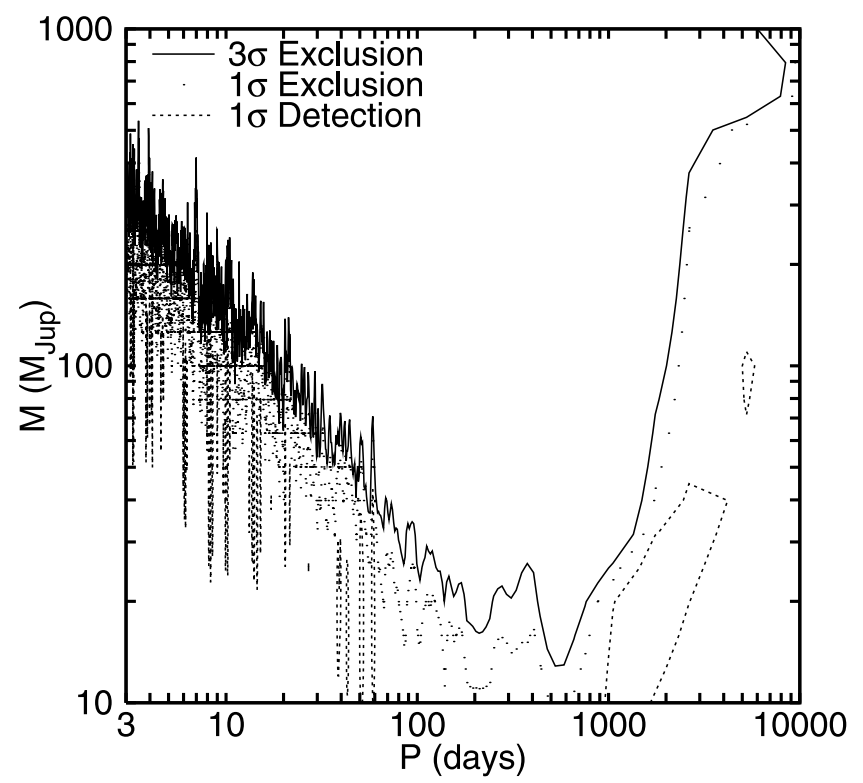

FIG. 10.-20 Persei mass-period phase space, by which a range of brown dwarf companions can be ruled out. Unfortunately, the PHASES observations do not yet have long enough time coverage to confirm the 1269 day period companion of Abt and Levy (1976). Owing to the far distance of the system and the higher mass stars, the PHASES observations are not particularly sensitive to planetary companions in this system. 


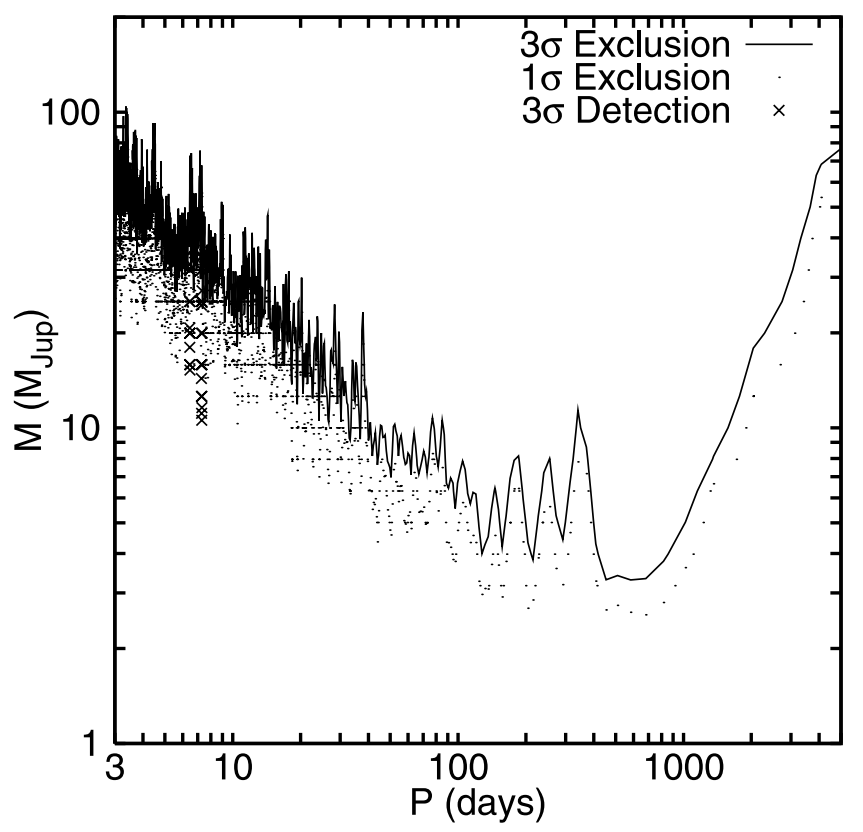

FIG. 11. $-\eta \mathrm{CrB}$ mass-period companion phase space, showing that planets as small as $3 M_{\mathrm{J}}$ are inconsistent with PHASES observations. A nearly $5 \sigma$ significant detection is found at short periods, but observational coverage does not yet allow the orbit to be strongly constrained; it is unclear whether this is merely a statistical fluke, perhaps related to the data sampling function (both dips are near 7 days, a natural sampling time for PHASES observations).

Detections are found for several different companion periods at the $1 \sigma$ detection level. The smallest value of $\chi_{\mathcal{B}}^{2}$ appears at the $2.9 \sigma$ level; as in the case of $\beta \mathrm{CrB}$, when the face-on and circular restrictions are lifted from the perturbation orbit, its parameters become poorly constrained. Abt \& Levy (1976) proposed the existence of a tertiary companion with period $P_{2}=1269 \pm$ 70 days; this periodicity is very close to the steep cutoff in the phase-space contour, which is a result of the finite span of PHASES observations, and at this time no conclusions are made about the existence of such a companion.

\section{0. $\eta$ CORONAE BOREALIS}

Seventeen PHASES observations of $\eta$ Coronae Borealis (2 CrB, HR 5727, HD 137107, ADS 9617) have been collected over a span of $T \sim 680$ days. As with 20 Per, $P_{1}$ and $e_{1}$ are fixed at values of $\sim 41.585 \mathrm{yr}$ and 0.2620 , determined by speckle interferometry (Mason et al. 1999), to lift strong covariances with $a_{1}$. From Hipparcos data, Söderhjelm (1999) determined a parallax of $53.5 \pm 0.9$ mas, a total mass of $2.41 M_{\odot}$, and a photometric mass ratio of 0.96 . From these, the tertiary orbit to $a_{2}$ conversion is made assuming a distance of $18.69 \mathrm{pc}$ and stellar mass of $1.18 M_{\odot}$ (the primary's mass is roughly $1.23 M_{\odot}$ and the tertiary companion limits are similar for primary and secondary). The mass-period grid is set up as in the case of $\delta$ Equ.

Companions as small as 3-4 $M_{\mathrm{J}}$ in orbits of $\sim 100-700$ days are inconsistent with the PHASES observations (see Fig. 11). As in the case of $\beta \mathrm{CrB}$, detection contours deeper than $3 \sigma$ appear (here at periods less than 10 days). The deepest of these is at the $4.8 \sigma$ level. Similarly to $\beta \mathrm{CrB}$, the data sampling does not allow all parameters of the perturbation orbit to be well constrained when the restrictions of face-on and circular orbits are lifted (in particular, $e_{2}$ is not constrained at all). This may be astrophysical in origin, but the current data cannot properly address the underlying orbit.

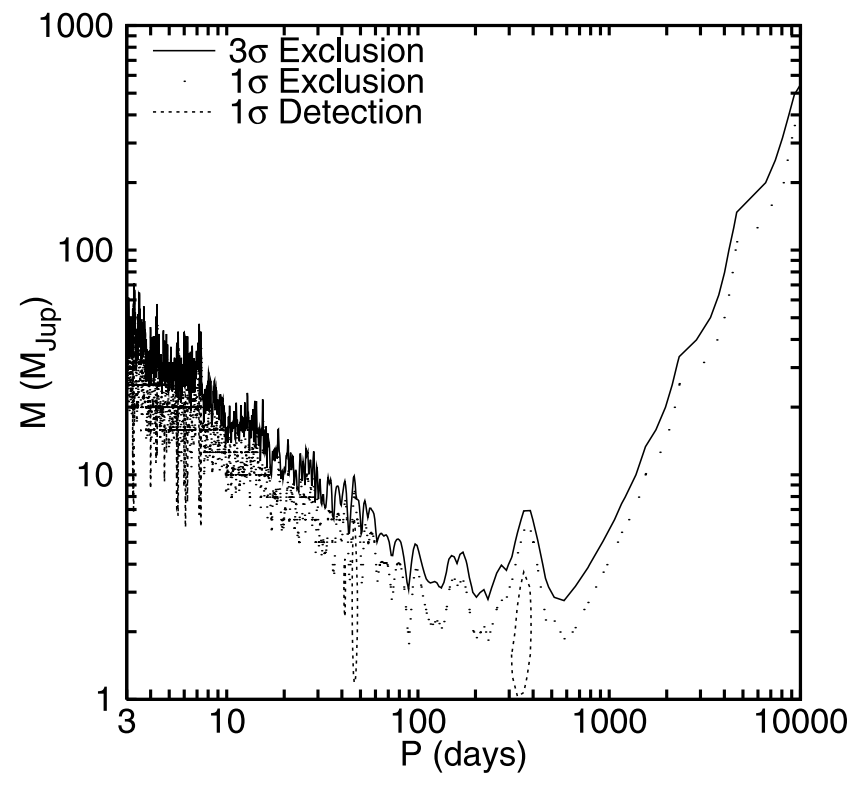

FIG. 12.-Contours in the companion mass-period phase space for HR 7162, showing that despite high noise levels from systematic processes, companions as small as $2 M_{\mathrm{J}}$ can be excluded in face-on orbits. After engineering improvements to remove these systematic noise sources, constraints will be placed on sub-Jupiter mass planets by second stage PHASES observations.

\section{HR 7162}

HR 7162 (HD 176051, ADS 11871) is a G0 V primary with K1 V secondary. This color difference appears to affect the PHASES data through the differential dispersion mechanism, because the excess scatter $\chi_{\mathcal{A}}^{2} / Z_{\mathcal{A}}$ is much larger than for other stars $\left(\sim 88 \sim 9.4^{2}\right)$. As with 20 Per and $\eta \mathrm{CrB}$, both $P_{1}$ and $e_{1}$ are fixed at values of $\sim 61.15 \mathrm{yr}$ and 0.258 , determined by Heintz (1994), to lift strong covariances with $a_{1}$. Over a span of $\sim 773$ days, 32 PHASES observations have been collected. The tertiary massto- $a_{2}$ conversion assumes a distance of $15 \mathrm{pc}$ and a star mass of $0.71 M_{\odot}$. These quantities are derived from the Hipparcos-based solution of Söderhjelm (1999), who found a parallax of $66.7 \pm$ 0.6 mas, a mass sum of $1.78 M_{\odot}$, and a photometric mass ratio of 0.67 (the primary mass corresponds to $1.07 M_{\odot}$ ). The massperiod grid is constructed as in the case of $\delta$ Equ. The smallest values of $\chi_{\mathcal{B}}^{2}$ correspond to a $2.7 \sigma$ detection. Companions as small as $2 M_{\mathrm{J}}$ are inconsistent with the PHASES data (see Fig. 12).

The physical properties of the HR 7162 binary (low-mass stars, close to Earth) make it an exciting candidate for an exoplanet search as very low mass companions can be detected for a given astrometric performance levels. Unfortunately, the PHASES excess scatter factor for this binary is currently much larger than that typical in other systems studied and appears to be random noise. This is likely due to the large color difference of the binary components, potentially leading to differential dispersion issues. It is anticipated that the current engineering improvements will benefit this system in particular, in which case it will be an extremely exciting study for the exoplanet search, with the ability to detect (or rule out) planets as small as a fifth of a Jupiter mass orbiting either star.

\section{CONCLUSIONS}

PHASES observations are able to exclude tertiary companions with masses as small as a few Jupiter masses in several binary systems. The phase-space searching algorithm correctly identifies the natures of two previously studied triple star systems and finds the correct periods for the short period pairs. While the 
number of systems that can currently be examined is too small to make strong conclusions about the frequency of giant planets in close binary systems, by the end of the PHASES program enough measurements will be made on all target systems to address this important question. Systematic effects currently limit the observed precision; if overcome, companions as small as a fifth of a Jupiter mass might be detected in favorable systems such as HR 7162.

PHASES benefits from the efforts of the PTI collaboration members who have each contributed to the development of an extremely reliable observational instrument. Without this outstanding engineering effort to produce a solid foundation, advanced phase-referencing techniques would not have been possible. We thank PTI's night assistant Kevin Rykoski for his efforts to maintain PTI in excellent condition and for operating PTI in phasereferencing mode every week. We thank Dimitri Pourbaix for helpful conversations about astrometric orbit fitting. Part of the work described in this paper was performed at the Jet Propulsion Laboratory under contract with the National Aeronautics and Space Administration. Interferometer data was obtained at the Palomar Observatory using the NASA Palomar Testbed Interferometer, supported by NASA contracts to the Jet Propulsion Laboratory. This research has made use of the Washington Double Star Catalog maintained at the US Naval Observatory. This research has made use of the Simbad database, operated at CDS, Strasbourg, France. M. W. M. acknowledges the MIT Earth, Atmospheric, and Planetary Sciences department for hosting him while this paper was written. B. F. L. acknowledges support from a Pappalardo Fellowship in Physics. PHASES is funded in part by the California Institute of Technology Astronomy Department, and by the National Aeronautics and Space Administration under grant NNG05GJ58G issued through the Terrestrial Planet Finder Foundation Science Program. This work was supported in part by the National Science Foundation through grants AST 0300096 and AST 0507590.
Abt, H. A., \& Levy, S. G. 1976, ApJS, 30, 273

Boden, A. F., et al. 1998, ApJ, 504, L39

Boss, A. P. 2000, ApJ, 536, L101

Chauvin, G., Lagrange, A., Udry, S., Fusco, T., Galland, F., Naef, D., Beuzit, J., \& Mayor, M. 2006, A\&A, 456, 1165

Colavita, M. M., et al. 1999, ApJ, 510, 505

Docobo, J. A., Tamazian, V. S., Balega, Y. Y., Blanco, J., Maximov, A. F., \& Vasyuk, V. A. 2001, A\&A, 366, 868

Dvorak, R., 1982, Oesterreichische Akad. Wissensch., Math.-natur., 191, 423

Harrison, E. R. 1977, Nature, 270, 324

Hartkopf, W. I., McAlister, H. A., \& Franz, O. G. 1989, AJ, 98, 1014

Hatzes, A. P., Cochran, W. D., Endl, M., McArthur, B., Paulson, D. B., Walker, G. A. H., Campbell, B. \& Yang, S. 2003, ApJ, 599, 1383

Heintz, W. D., 1994, AJ, 108, 2338

Kamper, K. W., McAlister, H. A., \& Hartkopf, W. I. 1990, AJ, 100, 239

Konacki, M. 2005, Nature, 436, 230

Lane, B. F., \& Muterspaugh, M. W. 2004, ApJ, 601, 1129

Lissauer, J. J. 1993, ARA\&A, 31, 129

Mason, B. D., Douglass, G. G., \& Hartkopf, W. I. 1999, AJ, 117, 1023

\section{REFERENCES}

Muterspaugh, M. W., Lane, B. F., Konacki, M., Burke, B. F., Colavita, M. M., Kulkarni, S. R. \& Shao, M. 2005, AJ, 130, 2866

. 2006a, A\&A, 446, 723

Muterspaugh, M. W., Lane, B. F., Konacki, M., Wiktorowicz, S., Burke, B. F., Colavita, M. M., Kulkarni, S. R., \& Shao, M. 2006b, ApJ, 636, 1020

Nelson, A. F. 2000, ApJ, 537, L65

Neubauer, F. J. 1944, ApJ, 99, 134

Pfahl, E. 2005, ApJ, 635, L89

Pfahl, E., \& Muterspaugh, M. 2006, ApJ, 652, 1694

Portegies Zwart, S. F., \& McMillan, S. L. W. 2005, ApJ, 633, L141

Press, W. H., Teukolsky, S. A., Vetterling, W. T., \& Flannery, B. P. 1992, Numerical Recipes in C: The Art of Scientific Computing (2nd ed.; Cambridge: Cambridge Univ. Press)

Queloz, D., et al. 2000, A\&A, 354, 99

Söderhjelm, S., 1999, A\&A, 341, 121

Tokovinin, A. A. 1984, Soviet Astron. Lett., 10, 121

Zucker, S., Mazeh, T., Santos, N. C., Udry, S., \& Mayor, M. 2004, A\&A, 426, 695 